\title{
AVALIAÇÃO DA ESTRUTURA ELETRÔNICA DA FASE MONOCLINICA DO ÓXIDO DE NIÓBIO COM BASE NO USO DE DIFERENTES FUNCIONAIS DE DENSIDADE
}

\author{
Kamila S. Ody ${ }^{a}$, João P. A. de Jesus ${ }^{a}$, Carlos E. Cava ${ }^{\mathrm{b}}$, Anderson R. Albuquerque ${ }^{\mathrm{c}}$, Ary S. Maia ${ }^{\mathrm{d}}$, Julio R. Sambrano e \\ Felipe A. La Porta ${ }^{\mathrm{a}, *,(1)}$ \\ aDepartamento de Química, Universidade Tecnológica Federal do Paraná, 86036-370 Londrina - PR, Brasil \\ 'Departamento de Engenharia de Materiais, Universidade Tecnológica Federal do Paraná, 86036-370 Londrina - PR, Brasil \\ 'Instituto de Química, Universidade Federal do Rio Grande do Norte, 59078-970 Natal - RN, Brasil \\ dNúcleo de Pesquisa e Extensão LACOM, Universidade Federal da Paraíba, 58051-900 João Pessoa - PB, Brasil \\ ${ }^{\mathrm{e}}$ Grupo de Modelagem e Simulação Molecular, Universidade Estadual Paulista,17033-360 Bauru - SP, Brasil
}

Recebido em 18/01/2021; aceito em 03/05/2021; publicado na web em 16/06/2021

\begin{abstract}
ASSESSMENT OF THE ELECTRONIC STRUCTURE OF THE MONOCLINIC PHASE OF NIOBIUM OXIDE BASED ON THE USE OF DIFFERENT DENSITY FUNCTIONALS. Niobium oxides, $\mathrm{Nb}_{2} \mathrm{O}_{5}$, are considered semiconductor materials with very attractive physical and chemical properties for applications in many areas, such as catalysis, sensors, medical, aerospace, etc. Especially, the characterization of $\mathrm{Nb}_{2} \mathrm{O}_{5}$-based nanostructures with monoclinic structure has received much attention in recent years. However, despite the great importance of this system, some of its fundamentals properties are still not fully understood. Hence, this work aims to apply the theoretical methodologies through Density Functional Theory (DFT) calculations in periodic models based on the use of different density functionals (like B1WC, B3PW, B3LYP, PBE0, PBESOL0, SOGGAXC, and WC1LYP) to investigate the physical and chemical properties of the monoclinic structure of $\mathrm{Nb}_{2} \mathrm{O}_{5}$. The band structures, energy bandgap, density of state, and vibrational properties, as well as order-disorder effects on the monoclinic structure of $\mathrm{Nb}_{2} \mathrm{O}_{5}$ are investigated in this study. Our theoretical results show a better agreement with experimental data for the B3LYP functional and hence lead to new perspectives on the deeper physicochemical understanding of the monoclinic $\mathrm{Nb}_{2} \mathrm{O}_{5}$. From these computational tools, it is possible to unravel the relations between structure and properties, which may contribute to the future development of new devices and applications based on these materials.
\end{abstract}

Keywords: $\mathrm{Nb}_{2} \mathrm{O}_{5}$; monoclinic; DFT; exchange-correlation; electronic structure.

\section{INTRODUÇÃO}

Nas últimas décadas, os nanomateriais a base do óxido de nióbio ganharam ênfase devido às suas promissoras aplicações industriais. ${ }^{1-4}$ Sendo o Brasil o maior depositário de reservas de nióbio (com aproximadamente $98 \%$ do total mundial), ${ }^{2}$ o Ministério de Minas e Energia tem fortemente recomendado a ampliação, bem como a disseminação da pesquisa científica e tecnológica, para explorar o desenvolvimento de novos materiais e produtos multifuncionais a base desse elemento estratégico. ${ }^{4}$ Diversos materiais baseados no nióbio - em suas diferentes formas e composições - têm sido aplicados com grande sucesso em diversos setores, como por exemplo, em equipamentos médicos e ópticos, na indústria aeroespacial, em dispositivos fotovoltaicos, catalisadores, sensores e em outras tecnologias avançadas..$^{1,2,5-9}$ Entretanto, apesar da sua grande relevância tecnológica, quando comparado aos outros metais de transição, a química do nióbio $(\mathrm{Nb})$ ainda está em sua plena infância. ${ }^{10}$

Deve-se notar que os óxidos de nióbio podem existir em diferentes estequiometrias, tais como o monóxido de nióbio $(\mathrm{NbO})$, dióxido de nióbio $\left(\mathrm{NbO}_{2}\right)$ e, como foco principal, o pentóxido de nióbio $\left(\mathrm{Nb}_{2} \mathrm{O}_{5}\right){ }^{1}$ Particularmente, o $\mathrm{NbO}$ é um sólido de coloração cinza que se cristaliza em uma típica estrutura cúbica e possui excelentes propriedades de condução elétrica. ${ }^{11,12}$ Por outro lado, o $\mathrm{NbO}_{2}$ é um sólido azul escuro, insolúvel em água, pode apresentar comportamento diamagnético e também é considerado como um forte agente redutor. ${ }^{1}$ Recentemente, nanopartículas de $\mathrm{NbO}_{2}$ foram aplicadas em baterias de íon de lítio. ${ }^{13,14}$ Do ponto de vista estrutural,

*e-mail: felipelaporta@utfpr.edu.br o $\mathrm{NbO}_{2}$ tem sido obtido em diferentes estruturas cristalinas: rutilo ( $\left.4_{2} / \mathrm{mnm}\right)$, ortorrômbica (Pbca), tetragonal (I4_1), triclínica (P1) e monoclínica $(\mathrm{C} 2 / \mathrm{m}) .^{12,15,16}$ Esse material exibe um bandgap indireto de aproximadamente $0,7 \mathrm{eV}$ e a natureza da sua transição metalisolante tem sido investigada, sendo geralmente atribuída a uma transição estrutural do tipo Peierls de segunda ordem. ${ }^{17,18}$ No entanto, é preciso atentar que suas propriedades físicas e químicas ainda não são completamente conhecidas.

Têm sido reportado na literatura que o $\mathrm{Nb}_{2} \mathrm{O}_{5}$, um material semicondutor de bandgap largo (do tipo " $n$ "), devido a sua grande energia de bandgap entre 3,1 e 5,3 eV. ${ }^{19}$ Em particular, esse material é facilmente acessível e apresenta uma grande estabilidade química, além de ser tipicamente um sólido branco insolúvel em água. ${ }^{19,20}$ Geralmente, possui uma estrutura cristalina formada de clusters octaédricos de $\left[\mathrm{NbO}_{6}\right]$, podendo ser distorcidos em diferentes graus e assim demonstrando outros sistemas cristalinos a curto e longo alcance, dependendo das condições de preparo e processamento. ${ }^{1,12,20}$ Em algumas fases, é possível encontrar diferentes estruturas de coordenação, tais como a heptacoordenada $\left[\mathrm{NbO}_{7}\right]$ e octacoordenada $\left[\mathrm{NbO}_{8}\right] .{ }^{1,12}$ Ainda assim, entre os diversos estudos sobre as estruturas polimórficas do $\mathrm{Nb}_{2} \mathrm{O}_{5}$, as três fases cristalográficas mais estudadas são: pseudohexagonal, ortorrômbica e monoclínica (Figura 1)..$^{21,22}$ Em condições ambientes, a fase monoclínica é a estrutura mais estável termodinamicamente, enquanto a fase pseudohexagonal é a menos estável..$^{22}$ Têm sido reportado também que o $\mathrm{Nb}_{2} \mathrm{O}_{5}$ apresenta uma estrutura ortorrômbica a aproximadamente $500{ }^{\circ} \mathrm{C}$ e, quando aquecido entre 700 a $800{ }^{\circ} \mathrm{C}$, é transformado em uma estrutura pseudohexagonal, ${ }^{22-24}$ enquanto que o aquecimento em temperaturas mais elevadas e na presença de agentes redutores (como o carbono) 


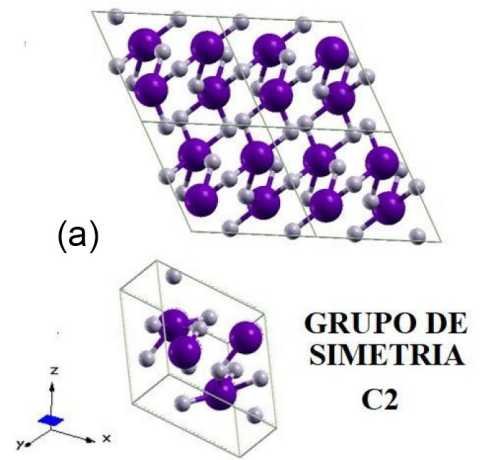

(b)
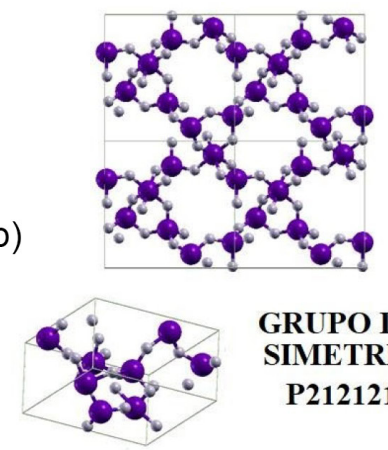

(c)

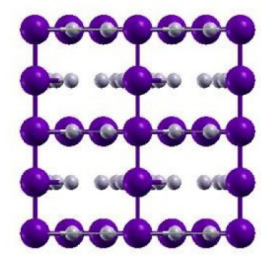

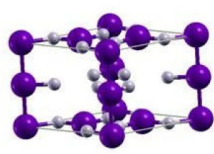

GRUPO DE SIMETRIA

$\mathbf{P 6} / \mathrm{m}$

Figura 1. Estruturas polimórficas do $\mathrm{Nb}_{2} \mathrm{O}_{5}($ a) monoclínica, (b) ortorrômbica e (c) pseudohexagonal

é responsável pela conversão total ou parcial do $\mathrm{Nb}_{2} \mathrm{O}_{5}$ em $\mathrm{NbO}_{2} \cdot{ }^{25}$ Contudo, é importante observar que essas temperaturas de transições cristalográficas ocorrem lentamente e são irreversíveis. ${ }^{24}$

Além de receber grande destaque no Brasil, o $\mathrm{Nb}_{2} \mathrm{O}_{5}$ mostra características que o tornam um material muito atrativo para diversas aplicações de alta performance. Isso é devido a sua boa estabilidade e resistência química em meio ácido e/ou básico. ${ }^{26}$ Entre suas diversas aplicações potenciais, pode-se destacar a fotocatálise heterogênea, utilizado tanto como fotocatalisador quanto adicionado a outros catalisadores; e na degradação de efluentes têxteis, corantes e fenol, em virtude da sua eficiência fotoativa. ${ }^{5,19,22,27-29}$ É aplicado também no desenvolvimento de baterias, células solares, diodos emissores de luz, ligas de aço (com função de atribuir melhores propriedades, principalmente em aços de alta resistência), automóveis e em superligas empregadas em turbinas de aeronaves, além de várias outras utilizações. ${ }^{1,19,29,30}$ No entanto, esse material avançado pode apresentar propriedades novas e interessantes quando utilizado na sua forma nanométrica. ${ }^{1,31}$

É certo que houve um grande avanço sobre o conhecimento das aplicações de nanomateriais e de algumas das suas propriedades fundamentais. ${ }^{12,15}$ Porém, ainda é necessário te um maior entendimento a respeito de suas estruturas nanométricas, com o intuito de pavimentar o desenvolvimento de novas tecnologias baseadas nesses materiais. Nesse cenário, as ferramentas computacionais vem tendo um enorme destaque no design de novos materiais avançados, tornando-se uma prática de pesquisa padrão no mundo inteiro. ${ }^{32,33}$ Sendo assim, diversas metodologias teóricas baseadas principalmente no formalismo da Teoria do Funcional da Densidade (DFT) vêm sendo desenvolvidas para acelerar o processo de descoberta e desenvolvimento da próxima geração de materiais avançados. ${ }^{34,35}$ Na prática, os cálculos de DFT envolvem o uso de aproximações amplamente conhecidas como "funcionais da densidade" para determinar, as propriedades e a energia de um sistema químico de interesse (considerando as contribuições de troca e correlação nesse tipo de cálculo). ${ }^{32,33,36-39}$

Portanto, o objetivo principal deste trabalho consiste em utilizar diversas metodologias teóricas baseadas em cálculos DFT - em condições periódicas - para o estudo da estrutura eletrônica e das propriedades físicas e químicas da fase monoclínica do $\mathrm{Nb}_{2} \mathrm{O}_{5}$. Com isso, por meio dos estudos de estruturas de bandas, do bandgap de energia e da densidade de estados (DOS), é possível desvendar as relações entre composição, estrutura, tamanho, morfologia e suas propriedades, de forma a contribuir para o desenvolvimento de novos materiais e dispositivos baseados no $\mathrm{Nb}_{2} \mathrm{O}_{5}$.

\section{METODOLOGIA}

No presente trabalho, os cálculos DFT foram realizados em condições periódicas utilizando do programa CRYSTAL $14 .{ }^{40}$ As funções de base foram escolhidas de acordo com as disponíveis na base de dados do software, sendo a TZVP 2018 para o Nb e a TZVP_2012 para o oxigênio. ${ }^{41,42}$ Os cálculos foram desenvolvidos a partir dos parâmetros de rede e de posições atômicas propostas na ficha ICSD $51176 .{ }^{43}$

Baseado no trabalho de Duarte et al. ${ }^{44}$ foi avaliado o desempenho de sete funcionais híbridos (B1WC, B3PW, B3LYP, PBE0, PBESOL0, SOGGAXC e WC1LYP) para os modelos de bulk ordenados e desordenados da estrutura monoclínica do $\mathrm{Nb}_{2} \mathrm{O}_{5}$. Os resultados obtidos foram comparados com outros estudos teóricos e experimentais encontrados na literatura, a fim de decidir o melhor funcional para descrever o sistema em questão. Embora as metodologias que englobam a DFT sejam conhecidas por apresentar boa robustez e correlação com os resultados experimentais, ${ }^{32,33}$ é reconhecido que tais métodos não levam em consideração os efeitos dispersivos (ou seja, interações de longo alcance de van der Waals). ${ }^{45-48}$ Sendo assim, o uso de correções de dispersão para o método DFT foi inicialmente proposto por Grimme. ${ }^{45-48}$ Mais especificamente, neste modelo a energia de dispersão pode ser simplesmente adicionada a energia de correlação-troca da DFT de acordo com a equação 1:

$$
E_{\text {disp }}^{D F T-D}=-\sum_{A B} \sum_{n=6,8,10 \ldots} s_{n} \frac{C_{n}^{A B}}{R_{A B}^{n}} f_{\text {damp }}\left(R_{A B}\right)
$$

Em que a soma é sobre todos os pares de elétrons do sistema de estudo, $C_{n}^{A B}$ o coeficiente de dispersão médio isotrópico de ordem $n$ $(n=6,8,10 \ldots)$ para o par de átomos $\mathrm{AB}, R_{A B}^{n}$ a distância entre núcleos, $s_{n}$ o fator de escala para ajustar o efeito repulsivo do funcional de densidade (uma vez que o nível B3LYP é conhecido por apresentar potenciais de interação puramente repulsivas para complexos fracamente ligados) e $f_{\text {damp }}$ como funções de amortecimento para evitar singularidades e contagem dupla dos efeitos de correlação para distâncias interatômicas baixas. ${ }^{45-50}$ Assim, neste estudo foram otimizados os parâmetros de rede e coordenadas atômicas para o raio de van der Waals do $\mathrm{Nb}\left(\mathrm{R}_{\mathrm{vdW}, \mathrm{Nb}}\right)$, variando em $\pm 25 \%$ os raios padrões propostos por Grimme, sendo $\mathrm{R}_{\mathrm{vdW}, \mathrm{Nb}}=1,639 \AA \AA^{47}$ Essa estratégia computacional, por sua vez, foi usada anteriormente pelo nosso grupo com grande sucesso no estudo de outros óxidos. ${ }^{50} \mathrm{~A}$ importância da descrição da influência do $\mathrm{R}_{\mathrm{vdw}, \mathrm{Nb}}$ é devido a característica de expressar com maior exatidão as propriedades do $\mathrm{Nb}_{2} \mathrm{O}_{5}$. Contudo, este parâmetro depende fortemente do ambiente químico e do tipo de estrutura cristalina. ${ }^{49,50}$ Portanto, em razão de sua grande relevância tecnológica, neste trabalho foi determinado o melhor valor de $\mathrm{R}_{\mathrm{vdW}, \mathrm{Nb}}$ para estrutura monoclínica do $\mathrm{Nb}_{2} \mathrm{O}_{5}$.

Ainda em questão ao polimorfo monoclínico, com intuito de descrever melhor a distorção do cluster ocasionada por efeitos estruturais no cristal, foram calculadas as distâncias médias de átomos de $\mathrm{Nb}$ e oxigênio ao centro do cluster, com o deslocamento do átomo de $\mathrm{Nb}$ em $0,3 \AA$, na direção $z$. Esses modelos podem ser 
úteis ao representar diferentes graus de ordem-desordem estrutural, possibilitando o maior entendimento químico de suas principais propriedades eletrônicas e espectroscopicas. ${ }^{51}$ Por fim, a estrutura eletrônica do $\mathrm{Nb}_{2} \mathrm{O}_{5}$ foi analisada e visualizada no programa gráfico XcrysDen. ${ }^{52}$

\section{RESULTADOS E DISCUSSÃO}

Uma decisão importante no desenvolvimento de um trabalho teórico com cálculos de DFT é a escolha do funcional a ser utilizado. Um funcional desejável deve atingir resultados satisfatório de exatidão que possibilitem descrever de maneira mais próxima possível à estrutura de um sistema e suas principais propriedades. Inúmeros trabalhos com essas finalidades são encontrados na literatura, ocasionalmente indicando o melhor funcional para um determinado sistema de interesse. ${ }^{44,53-55} \mathrm{~A}$ importância dessa prática se deve ao fato de alguns funcionais descreverem melhor as estruturas, mas não os parâmetros eletrônicos (tais como o bandgap), citando, por exemplo, os funcionais GGA e LDA. Em contrapartida, outros se comportam da forma inversa ao descreverem adequadamente o bandgap, enquanto superestimam os parâmetros de rede. ${ }^{44,56}$ Assim, nenhum funcional é capaz de simular com exatidão todos os detalhes e propriedades de um sistema alvo, sejam em termos estruturais, eletrônicos ou vibracionais, por exemplo.

Zibrov et $a l .{ }^{43}$ reportaram os parâmetros de rede experimentais para a fase monoclínica do $\mathrm{Nb}_{2} \mathrm{O}_{5}(a=5,2195 \AA$, $b=4,6995 \AA$ e $c$ $=5,9285 \AA$ ). Esses parâmetros estruturais foram comparados com nossos resultados obtidos e outros trabalhos teóricos encontrados na literatura, sendo descritos na Tabela 1 . Deve-se enfatizar que a estrutura monoclínica é a única que apresenta coordenação $\left[\mathrm{NbO}_{7}\right]$ entre todas reportadas. Portanto, essa característica peculiar pode ser considerada como uma das motivações para o desenvolvimento do presente estudo.

A Tabela 1 descreve os parâmetros de rede $(a, b$ e $c)$; os valores do ângulo $(\beta)$ e o volume da célula $(V)$ calculados para a estrutura monoclínica do $\mathrm{Nb}_{2} \mathrm{O}_{5}$. Uma análise desses resultados revela que os valores dos parâmetros experimentais $a$ e $b$ foram superestimados para todos os funcionais estudados, com exceção do SOGGAXC, tendo um valor $0,0738 \AA$ menor em comparação ao parâmetro $b$ experimental. Entretanto, os parâmetros $c$ foram subestimados para todos os funcionais, demonstrando valores menores que variam de $0,1352 \AA$ (B3LYP) a $0,2125 \AA$ (PBESOL0) em relação ao experimental. Também foi observado que para todos os funcionais o valor de $\beta$ estava acima de $90^{\circ}$ de acordo com a estrutura monoclínica; superestimando o resultado experimental com acréscimos variando de $5,55^{\circ}$ (SOGGAXC) a 8,37 (B3LYP). Já a diferença do volume da célula foi o fator de maior distinção entre os dados experimentais e os calculados por meio de diferentes funcionais, sendo superestimados e alternando de $2,60 \AA^{3}$ (SOGGAXC) a 20,42 $\AA^{3}$ (B3PW), como ilustrado na Figura 2 (a).

De maneira geral, o volume da célula unitária é influenciado diretamente pela presença de defeitos ${ }^{57}$ Quando a estrutura da célula é otimizada, o resultado é um sistema perfeito, portanto, o volume simulado tende a ser geralmente maior que o de uma célula unitária real. Essa tendência está em bom acordo com nossos resultados. Com isso, o funcional que estimou as propriedades estruturais da célula unitária de $\mathrm{Nb}_{2} \mathrm{O}_{5}$ mais próximas da realidade foi o SOGGAXC, enquanto as mais distantes foi o B3PW.

No design de novos materiais avançados, um dos parâmetros mais críticos é a energia de bandgap $\left(E_{\text {gap }}\right)$. $^{32,33} \mathrm{Na}$ literatura, o bandgap reportado experimentalmente para a fase monoclínica é de $3,79 \mathrm{eV}$, padrão que será usado na comparação dos resultados e como base

Tabela 1. Valores dos parâmetros estruturais da estrutura monoclínica e volumes das células calculadas com os diferentes funcionais

\begin{tabular}{|c|c|c|c|c|c|c|}
\hline Funcionais & $a(\AA)$ & $b(\AA)$ & $c(\AA)$ & $\alpha=\gamma\left({ }^{\circ}\right)$ & $\beta\left(^{\circ}\right)$ & $V\left(\AA^{3}\right)$ \\
\hline B1WC & 5,84 & 4,90 & 5,74 & 90 & 115,85 & 147,78 \\
\hline B3LYP & 6,01 & 5,10 & 5,79 & 90 & 116,93 & 153,98 \\
\hline B3PW & 5,94 & 5,04 & 5,76 & 90 & 116,72 & 158,27 \\
\hline PBE0 & 5,91 & 5,01 & 5,74 & 90 & 116,56 & 152,28 \\
\hline PBESOL10 & 5,82 & 4,90 & 5,72 & 90 & 115,91 & 146,64 \\
\hline SOGGAXC & 5,82 & 4,59 & 5,76 & 90 & 114,11 & 140,45 \\
\hline WC1LYP & 5,92 & 5,02 & 5,77 & 90 & 116,32 & 153,70 \\
\hline Jain A. et al. $(2013)^{16}$ & 6,18 & 5,25 & 5,85 & 90 & 118,62 & 166,60 \\
\hline Zibrov et al. $(1998)^{43}$ & 5,22 & 4,70 & 5,93 & 90 & 108,56 & 137,85 \\
\hline
\end{tabular}
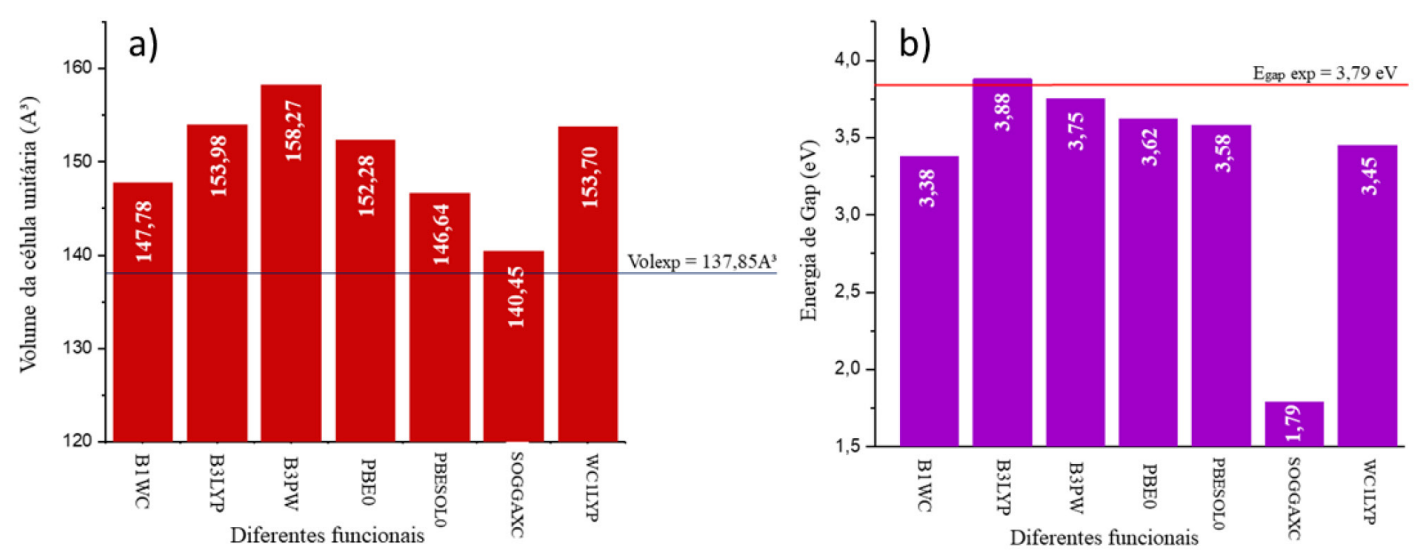

Figura 2. Comparação entre os valores de (a) volume e (b) energia de bandgap calculados utilizando de diferentes funcionais para a estrutura monoclínica do $\mathrm{Nb}_{2} \mathrm{O}_{5}$ 
de análise na escolha do funcional ideal. ${ }^{19}$ Com a finalidade de facilitar a discussão a seguir, os resultados que serão apresentados foram organizados na Figura 2 (b). Complementando o estudo das propriedades eletrônicas do sistema, na Figura 3 estão ilustradas as estruturas de bandas simuladas para os diferentes funcionais utilizados neste trabalho. $\mathrm{O}$ caminho de simetria da zona de Brillouin para a célula monoclínica primitiva foi definido por G-Y-Z-T-S-R ${ }^{58} \mathrm{~A}$ análise dos resultados de estrutura de bandas do $\mathrm{Nb}_{2} \mathrm{O}_{5}$ evidencia uma transição indireta para todos os casos investigados. Porém, ao checar a estrutura de bandas obtida pelo funcional SOGGAXC (Figura 3 (f)), é notado um bandgap estreito em comparação aos demais, também indireto $(\mathrm{R}-\mathrm{Y})$ e igual a $1,79 \mathrm{eV}$. Esse valor é muito abaixo do esperado para a estrutura monoclínica do $\mathrm{Nb}_{2} \mathrm{O}_{5}$.
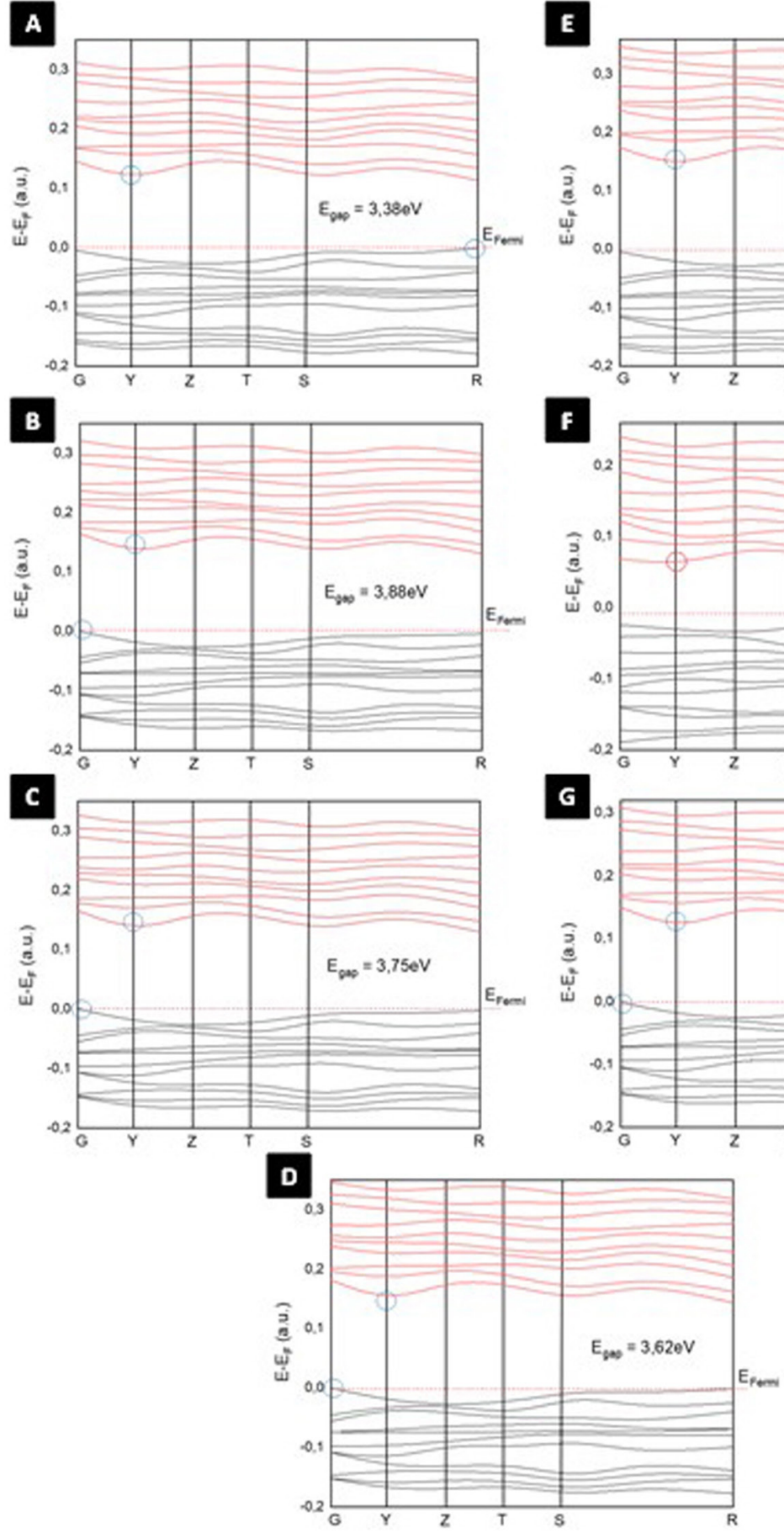

Figura 3. Estruturas de bandas simuladas para o bulk com diferentes funcionais: a) B1WC, b) B3LYP, c) B3PW, d) PBEO, e) PBESOLO,f) SOGGAXC e g) WCLYP

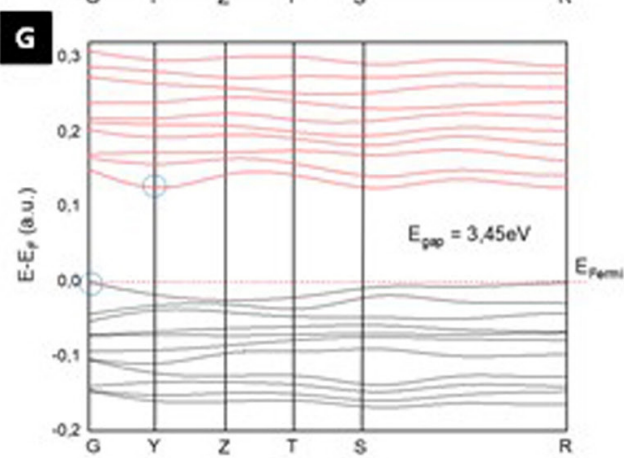

Por meio do comportamento visto na estrutura de bandas, é possível afirmar que o funcional SOGGAXC não é o mais recomendado para o estudo das propriedades eletrônicas do $\mathrm{Nb}_{2} \mathrm{O}_{5}$. Assim, como o bandgap é o principal parâmetro no desenvolvimento de novos materiais, podemos observar que as simulações por meio do funcional B3LYP é a metodologia mais adequada no estudo das propriedades eletrônicas do sistema em questão.

A Figura 4 mostra as densidades de estados (DOS) para o modelo ordenado do $\mathrm{Nb}_{2} \mathrm{O}_{5}$. Como resultado, a análise do DOS revela que a banda de valência (BV) do sistema é constituída principalmente por orbitais $2 p$ do oxigênio, enquanto que a banda de condução (BC) é majoritariamente formada por orbitais $4 d$ do $\mathrm{Nb}$. Esses dados são consistentes com estudos teórico-experimental prévios. ${ }^{17}$ No entanto,

E

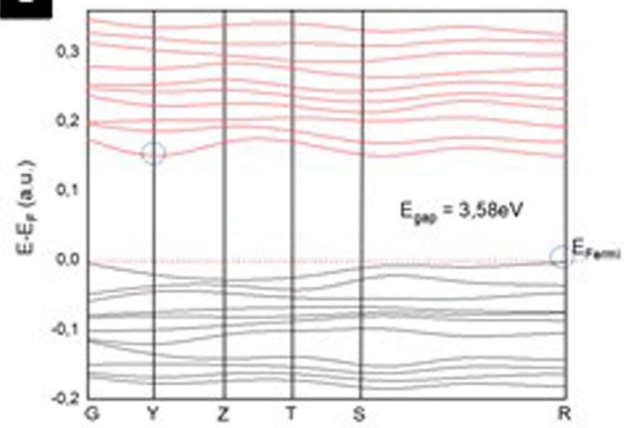

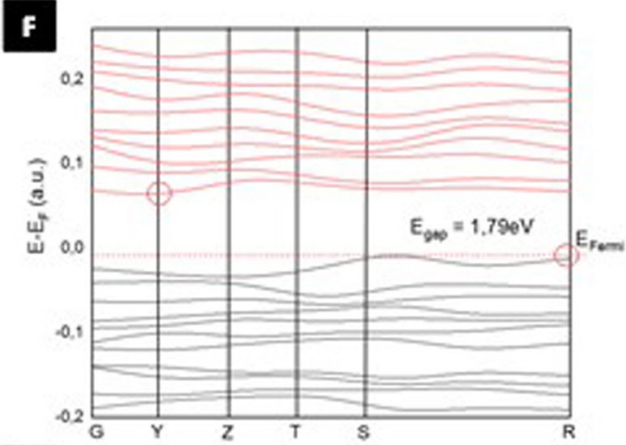



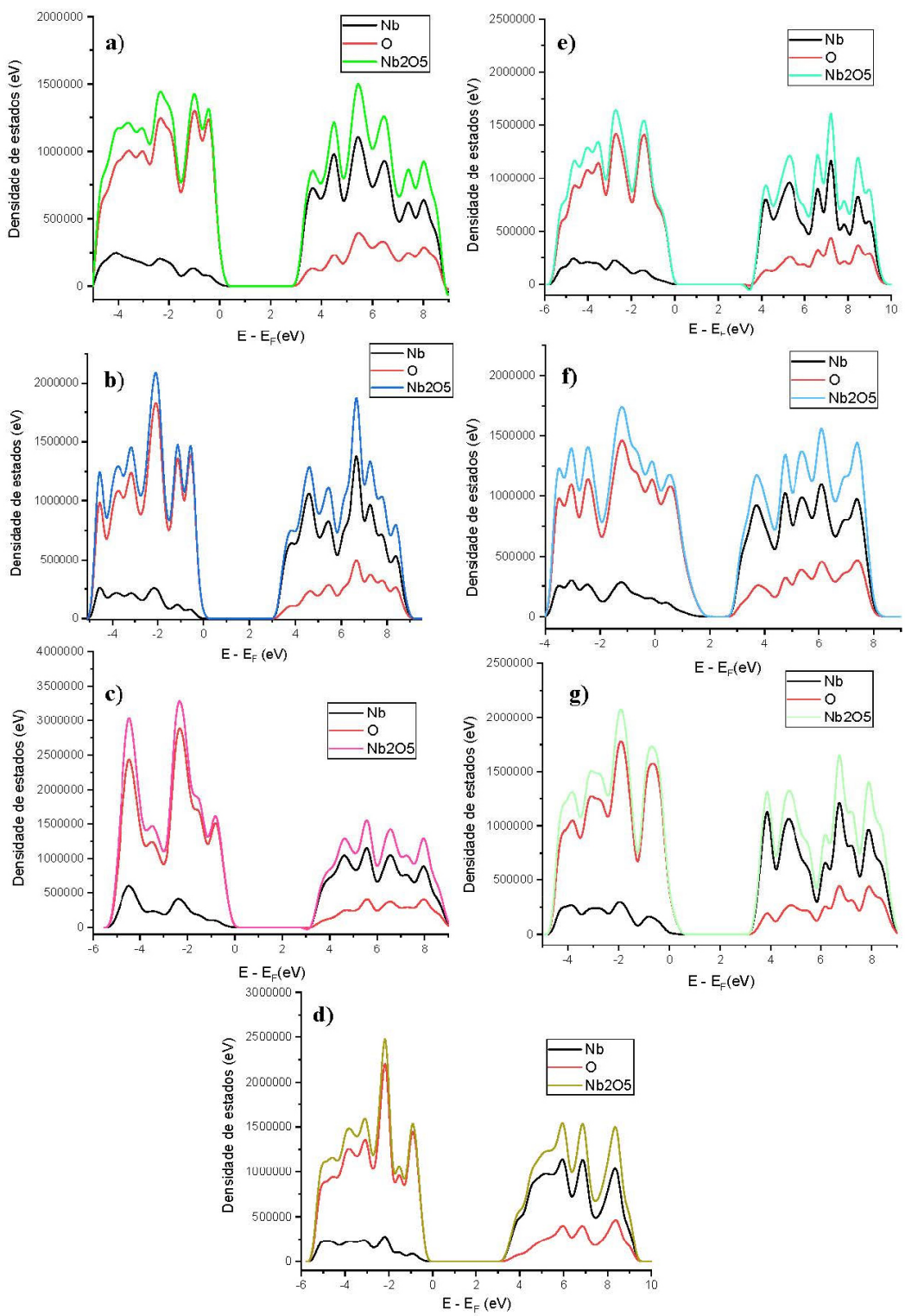

Figura 4. Densidades de estados simuladas para o bulk do $\mathrm{Nb}_{2} \mathrm{O}_{5}$ por meio de diferentes funcionais: a) B1WC, b) B3LYP, c) B3PW, d) PBEO, e) PBESOLO, f) SOGGAXC e g) WCILYP

está evidente que o perfil do DOS é ligeiramente diferente para todos os casos analisados, devido ao fato das estruturas otimizadas apresentarem diferentes parâmetros estruturais, como apresentados na Tabela 1.

Após a escolha do melhor funcional com base na análise das propriedades eletrônicas, foi simulado o espectro Raman para a estrutura monoclínica do $\mathrm{Nb}_{2} \mathrm{O}_{5}$. Na Figura 5 é apresentada a comparação entre as posições dos modos Raman ativos obtidos no espectro teórico com o funcional B3LYP e as posições experimentais. Experimentalmente, os modos Raman ativos da estrutura monoclínica do $\mathrm{Nb}_{2} \mathrm{O}_{5}$ são localizados em torno de 78, 127, 227, 307, 521, 642, 689 e $843 \mathrm{~cm}^{-1}$, e na faixa entre 500 a $1100 \mathrm{~cm}^{-1}$ são encontradas as bandas das vibrações do oxigênio. ${ }^{29,59}$ Os picos presentes na região entre 400 a $800 \mathrm{~cm}^{-1}$ são atribuídos aos alongamentos simétricos e antissimétricos das ligações $\mathrm{Nb}-\mathrm{O}-\mathrm{Nb}^{60}{ }^{60}$ Utilizando do funcional B3LYP, foram alcançados resultados bastante coerentes com os dados experimentais desse polimorfo, com modos Raman ativos nas mesmas posições em ambos os espectros.

No entanto, sabemos que as propriedades fundamentais dos materiais semicondutores dependem fortemente de seus defeitos. ${ }^{32}$
Por esse motivo, foram realizados cálculos da estrutura desordenada do $\mathrm{Nb}_{2} \mathrm{O}_{5}$, considerando um deslocamento dos átomos de $\mathrm{Nb}$ igual a 0,3 Å na direção $z$. Uma análise comparativa dos modelos ordenado e desordenado da estrutura monoclínica do $\mathrm{Nb}_{2} \mathrm{O}_{5}$ é apresentada na Figura 6. A energia de bandgap do bulk desordenado decai para $3,82 \mathrm{eV}$, mais próxima do valor experimental de $3,79 \mathrm{eV} .{ }^{19}$ Somandose a isso, os mapas de densidade eletrônica para os modelos ordenado e desordenado do bulk são exibidos na Figura 7 (c,d) em diferentes perspectivas. Esses resultados reforçam a existência de dois ambientes de coordenação $\left(\left[\mathrm{NbO}_{6}\right]\right.$ e $\left.\left[\mathrm{NbO}_{7}\right]\right)$ para a fase monoclínica, podendo auxiliar no entendimento da estrutura local do $\mathrm{Nb}_{2} \mathrm{O}_{5}$ e sendo a origem das suas principais propriedades.

Por outro lado, é bem conhecido que as interações não-covalentes desempenham um importante papel no estudo das propriedades desses cristais. Diante dessa consideração, foram executados cálculos considerando os efeitos dispersivos, conforme descrito na metodologia. Para os cálculos de otimização adicionando a correção do parâmetro de Grimme, uma pequena variação no $\mathrm{R}_{\mathrm{vdW}}$ ocasiona em uma ligeira alteração nos parâmetros de rede e, por consequência, na energia total, no bandgap e em outras propriedades. ${ }^{49,50} \mathrm{O} \mathrm{R}_{\mathrm{vdW}}$ do oxigênio já está 


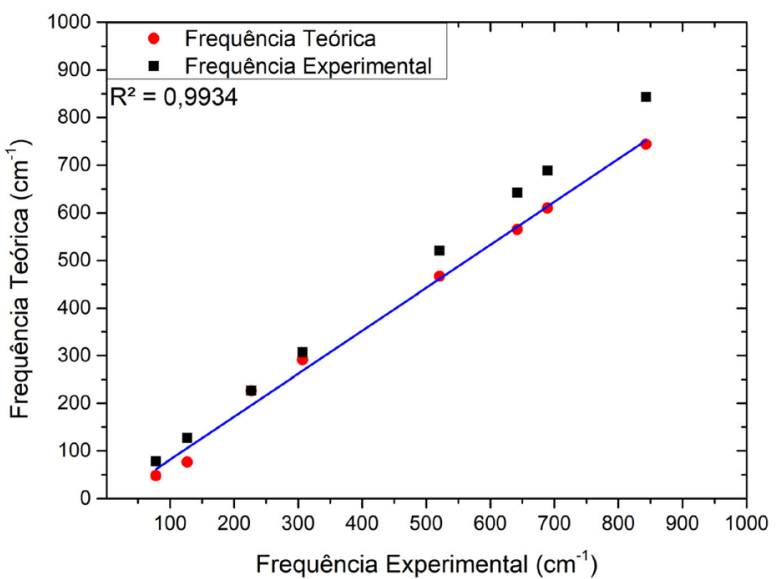

Figura 5. Comparação entre as frequências vibracionais do espectro Raman experimental e teórico $(B 3 L Y P)$
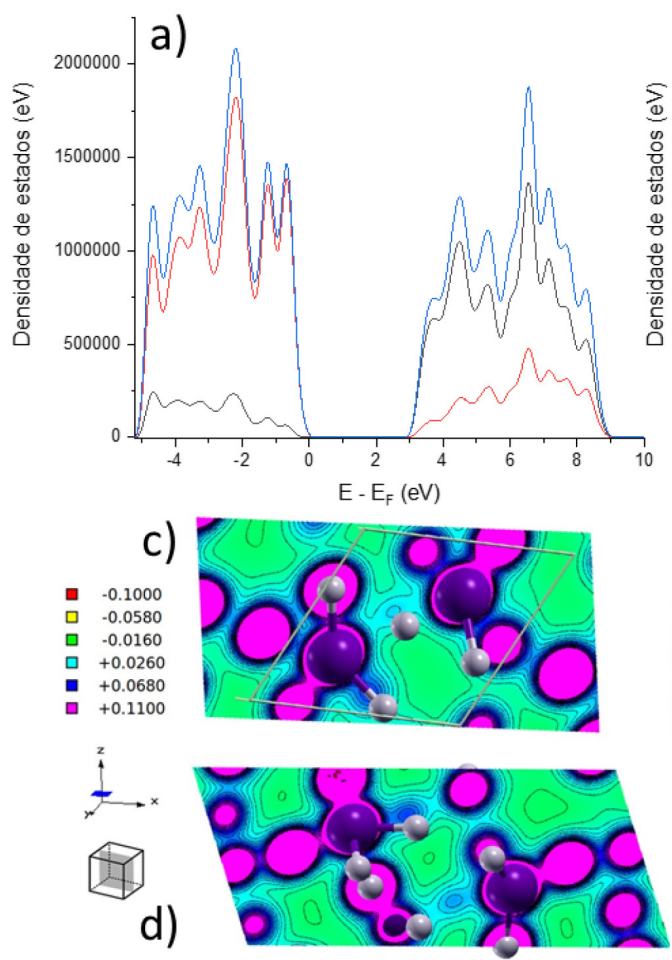

Figura 6. Densidades de estados simuladas por orbital para o bulk a) ordenado e b) desordenado. Mapas de densidade de carga para estrutura c) ordenada e d) desordenada (horizontal)

a)

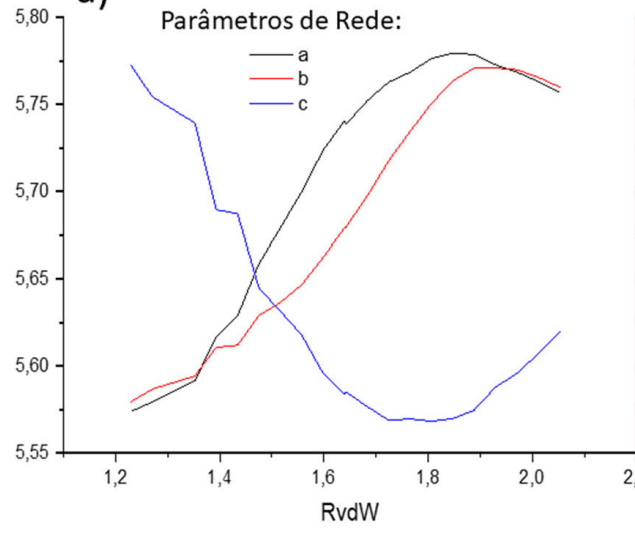

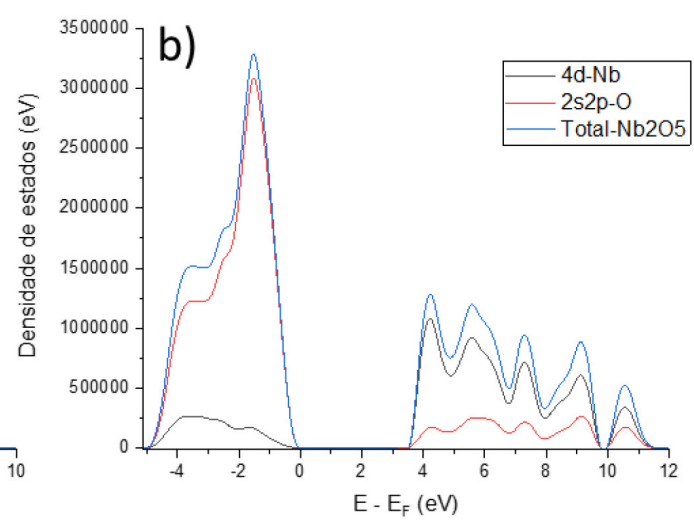
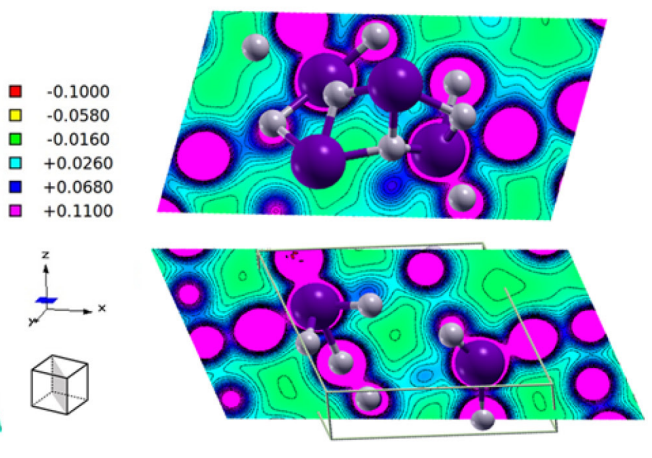

consolidado e parametrizado para diferentes óxidos, com valor igual a $1,342 \AA \mathrm{e} \mathrm{C}_{6}$ de $0,700 \cdot{ }^{47,49}$ Com isso, os parâmetros de rede otimizados em função da variação do $R_{v d W, N b}$ são mostrados na Figura 7 (a).

Em comparação com os parâmetros de rede obtidos experimentalmente ( $a=5,2195 \AA, b=4,6995 \AA$ e $c=5,9285 \AA$ ), é observado que o parâmetro $a$ teórico difere significativamente do experimental para o intervalo de raios considerados, enquanto que o parâmetro $b$ é mais coerente com o valor real à medida o $\mathrm{R}_{\mathrm{vdw}, \mathrm{Nb}}$ aumenta. ${ }^{43} \mathrm{O}$ parâmetro $c$ se aproxima do experimental, mesmo apresentando uma pequena diferença nos valores intermediários do $\mathrm{R}_{\mathrm{vdW,Nb}}$. A diminuição do parâmetro $c$ é acompanhada pelo aumento de $a$ e $b$ conforme a variação do $\mathrm{R}_{\mathrm{vdW}, \mathrm{N} b}$, evidenciando um predomínio experimental de distorções na direção do parâmetro $b$ e levando a um acréscimo das repulsões na direção de $a$ e $c$. Na posição em que o parâmetro independente $b$ é semelhante ao valor experimental, o $\mathrm{R}_{\mathrm{vdW.Nb}}$ é igual a $1,929 \AA \AA$ e os parâmetros internos otimizados são $a=5,7729 \AA, b=4,6961 \AA$ e $c=6,1287 \AA$. Dessa forma, o parâmetro $b$ $+0.1100$

b)
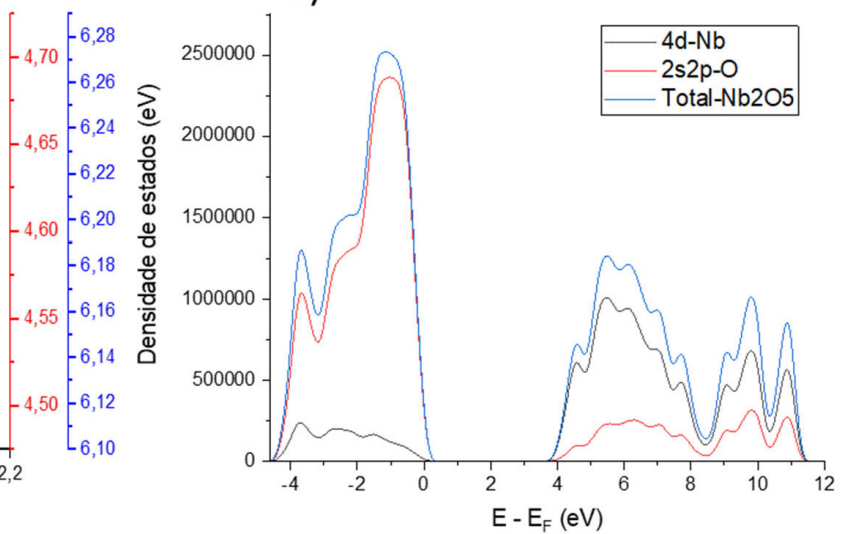

Figura 7. a) Parâmetros de rede em função da variação do raio de van der Waals para o $\mathrm{Nb}$ e b) densidade de estados simulada por orbital para o bulk monoclínico com parâmetro $R_{v d W, N b}=1,929$ 
é próximo ao experimental, variando em cerca de $0,0034 \AA$ A Também é visto uma repulsão esperada para os parâmetros $a$ e $c$, tendo alterações de, respectivamente, 0,5534 ̊ e 0,2002 A. A Figura 7 (b) mostra os resultados obtidos para a DOS considerando o $R_{\mathrm{vdWNb}}=1,929 \AA$ durante a otimização. De acordo os dados, podemos estimar que a energia de bandgap é igual a 3,76 eV, número mais consistente com o valor experimental. ${ }^{19}$ Assim, a metodologia proposta para incluir o $\mathrm{R}_{\mathrm{vdW}}$ do $\mathrm{Nb}$ nos cálculos computacionais se demonstrou eficiente $\mathrm{e}$ em concordância com o valor reportado para a energia de bandgap, alcançando uma melhor descrição dos parâmetros eletrônicos do polimorfo monoclínico do $\mathrm{Nb}_{2} \mathrm{O}_{5}$.

\section{CONCLUSÕES}

Neste estudo, a performance dos funcionais B1WC, B3PW, B3LYP, PBE0, PBESOL0, SOGGAXC e WC1LYP foi avaliada com fins de investigar a estrutura eletrônica e as propriedades espectroscópicas da fase monoclínica do $\mathrm{Nb}_{2} \mathrm{O}_{5}$. De acordo com nossos resultados, o funcional B3LYP se demonstrou o mais recomendado para descrever esse sistema. A simulação da estrutura desordenada do $\mathrm{Nb}_{2} \mathrm{O}_{5}$ sugeriu que a diminuição no valor do bandgap é devido a formação de dois ambientes de coordenação $\left(\left[\mathrm{NbO}_{6}\right]\right.$ e $\left.\left[\mathrm{NbO}_{7}\right]\right)$, contribuindo para um melhor entendimento de seu comportamento químico. Além disso, neste estudo também foi otimizado o $\mathrm{R}_{\mathrm{vdW}, \mathrm{Nb}}(1,929 \AA)$ para a fase monoclínica do $\mathrm{Nb}_{2} \mathrm{O}_{5}$. Assim, podemos concluir que o uso de efeitos dispersivos contribui para uma boa descrição dos parâmetros estruturais e eletrônicos do $\mathrm{Nb}_{2} \mathrm{O}_{5}$, em consonância com os resultados experimentais. Finalmente, é esperado que o presente trabalho possa contribuir para os estudos teóricos posteriores baseados no formalismo da DFT e que tenham como foco o sistema $\mathrm{Nb}_{2} \mathrm{O}_{5}$.

\section{AGRADECIMENTOS}

Os autores agradecem às agências de fomento brasileira (CNPq; CAPES; e FAPESP) e à UTFPR pelo apoio financeiro e pela bolsa concedida para realização deste trabalho.

\section{REFERÊNCIAS}

1. Lopes, O. F.; De Mendonça, V. R.; Silva, F. B. F.; Paris, E. C.; Ribeiro, C.; Quim. Nova 2015, 38, 106.

2. Lima, J. M. G. de; Relatório Técnico 20 - Perfil da Mineração do Nióbio; 2010.

3. Silberglitt, R.; Bartis, J.; Chow, B.; An, D.; Brady, K.; Critical Materials - Present Danger to US Manufacturing; 2013.

4. Brasil; Ministério de Minas e Energia; Departamento Nacional de Produção Mineral; DNPM. Nióbio; Brasília, 2009.

5. Nowak, I.; Ziolek, M.; Chem. Rev. 1999, 99, 3603.

6. Dinu, M.; Braic, L.; Padmanabhan, S. C.; Morris, M. A.; Titorencu, I.; Pruna, V.; Parau, A.; Romanchikova, N.; Petrik, L. F.; Vladescu, A.; J. Mech. Behav. Biomed. Mater. 2020, 103, 103582.

7. Yang, M.; Zhao, X.; Zheng, S.; Liu, X.; Jin, B.; Li, H.; Gan, Y.; J. Electroanal. Chem. 2017, 791, 17.

8. Pereira-Maia, E. C.; Souza, I. P.; Nunes, K. J. R. C.; Castro, A. A.; Ramalho, T. C.; Steffler, F.; Duarte, H. A.; Pacheli, A.; Chagas, P.; Oliveira, L. C. A.; RSC Adv. 2018, 8, 10310.

9. Rani, R. A.; Zoolfakar, A. S.; Ou, J. Z.; Field, M. R.; Austin, M.; Kalantar-zadeh, K.; Sens. Actuators, B 2013, 176, 149.

10. Bruziquesi, C.; Balena, J.; Pereira, M.; Silva, A.; Oliveira, L.; Quim. Nova 2019, 42, 1184.

11. Li, J.; Liu, W.-W.; Zhou, H.-M.; Liu, Z.-Z.; Chen, B.-R.; Sun, W.-J.; Rare Met. 2018, 37, 118.
12. Nico, C.; Monteiro, T.; Graça, M. P. F.; Prog. Mater. Sci. 2016, 80, 1.

13. Asfaw, H. D.; Tai, C.-W.; Nyholm, L.; Edström, K.; ChemNanoMat 2017, 3, 646.

14. Ji, Q.; Gao, X.; Zhang, Q.; Jin, L.; Wang, D.; Xia, Y.; Yin, S.; Xia, S.; Hohn, N.; Zuo, X.; Wang, X.; Xie, S.; Xu, Z.; Ma, L.; Chen, L.; Chen, G. Z.; Zhu, J.; Hu, B.; Müller-Buschbaum, P.; Bruce, P. G.; Cheng, Y.; Adv. Funct. Mater. 2019, 29, 1904961.

15. Jacob, K. T.; Shekhar, C.; Vinay, M.; Waseda, Y.; J. Chem. Eng. Data 2010, 55,4854 .

16. Jain, A.; Ong, S. P.; Hautier, G.; Chen, W.; Richards, W. D.; Dacek, S.; Cholia, S.; Gunter, D.; Skinner, D.; Ceder, G.; Persson, K. A.; APL Mater. 2013, 1, 011002.

17. O'Hara, A.; Demkov, A. A.; Phys. Rev. B 2015, 91, 094305.

18. Weibin, Z.; Weidong, W.; Xueming, W.; Xinlu, C.; Dawei, Y.; Changle, S.; Liping, P.; Yuying, W.; Li, B.; Surf. Interface Anal. 2013, 45, 1206.

19. Rani, R. A.; Zoolfakar, A. S.; O’Mullane, A. P.; Austin, M. W.; KalantarZadeh, K.; J. Mater. Chem. A 2014, 2, 15683.

20. Ko, E. I.; Weissman, J. G.; Catal. Today 1990, 8, 27.

21. Dash, J. K.; Chen, L.; Topka, M. R.; Dinolfo, P. H.; Zhang, L. H.; Kisslinger, K.; Lu, T.-M.; Wang, G.-C.; RSC Adv. 2015, 5, 36129.

22. Zhao, Y.; Zhou, X.; Ye, L.; Chi Edman Tsang, S.; Nano Rev. 2012, 3, 17631.

23. Rezende, C. C.; Neto, J. L.; Silva, A. C.; Lima, V. M.; Pereira, M. C.; Oliveira, L. C. A.; Catal. Commun. 2012, 26, 209.

24. Lopes, I. S.; Dissertação de Mestrado, Universidade Federal Fluminense, Brasil, 2003.

25. Kong, L.; Zhang, C.; Wang, J.; Qiao, W.; Ling, L.; Long, D.; Sci. Rep. 2016, 6, 21177.

26. Avellaneda, C. O.; Aegerter, M. A.; Pawlicka, A.; Quim. Nova 1998, 21, 365.

27. Santos, A. P. F.; Dissertação de Mestrado, Universidade Federal do Mato Grosso do Sul, Brasil, 2012.

28. Santana, V. S.; Machado, N. R. C. F.; Acta Sci. Technol. 2006, 28.

29. Raba, A. M.; Bautista-Ruíz, J.; Joya, M. R.; Mater. Res. 2016, $19,1381$.

30. He, J.; Hu, Y.; Wang, Z.; Lu, W.; Yang, S.; Wu, G.; Wang, Y.; Wang, S.; Gu, H.; Wang, J.; J. Mater. Chem. C 2014, 2, 8185.

31. Silva, F. O.; Viol, L. C. de S.; Ferreira, D. L.; Alves, J. L. A.; Schiavon, M. A.; Quim. Nova 2010, 33, 1933.

32. Recent Advances in Complex Functional Materials; Longo, E., La Porta, F. de A., eds.; Springer International Publishing: Cham, 2017.

33. Emerging Research in Science and Engineering Based on Advanced Experimental and Computational Strategies; La Porta, F. de A., Taft, C. A., eds.; Engineering Materials; Springer International Publishing: Cham, 2020.

34. Hohenberg, P.; Kohn, W.; Phys. Rev. 1964, 136, B864.

35. Kohn, W.; Sham, L. J.; Phys. Rev. 1965, 140, A1133.

36. Neugebauer, J.; Hickel, T.; Wiley Interdiscip. Rev. Comput. Mol. Sci. 2013, 3, 438.

37. Mardirossian, N.; Head-Gordon, M.; Mol. Phys. 2017, 115, 2315.

38. Capelle, K.; Braz. J. Phys. 2006, 36, 1318.

39. Alberi, K.; Nardelli, M. B.; Zakutayev, A.; Mitas, L.; Curtarolo, S.; Jain, A.; Fornari, M.; Marzari, N.; Takeuchi, I.; Green, M. L.; Kanatzidis, M.; Toney, M. F.; Butenko, S.; Meredig, B.; Lany, S.; Kattner, U.; Davydov, A.; Toberer, E. S.; Stevanovic, V.; Walsh, A.; Park, N.-G.; Aspuru-Guzik, A.; Tabor, D. P.; Nelson, J.; Murphy, J.; Setlur, A.; Gregoire, J.; Li, H.; Xiao, R.; Ludwig, A.; Martin, L. W.; Rappe, A. M.; Wei, S.-H.; Perkins, J.; J. Phys. D. Appl. Phys. 2019, 52, 013001.

40. Dovesi, R.; Orlando, R.; Civalleri, B.; Roetti, C.; Saunders, V. R.; Zicovich-Wilson, C. M.; Z. Kristallogr. - Cryst. Mater. 2005, 220.

41. Laun, J.; Vilela Oliveira, D.; Bredow, T.; J. Comput. Chem. 2018, 39, 1285.

42. Peintinger, M. F.; Oliveira, D. V.; Bredow, T.; J. Comput. Chem. 2013, 34,451 . 
43. Zibrov, I. P.; Filonenko, V. P.; Werner, P.-E.; Marinder, B.-O.; Sundberg, M.; J. Solid State Chem. 1998, 141, 205.

44. Duarte, T. M.; Buzolin, P. G. C.; Santos, I. M. G.; Longo, E.; Sambrano, J. R.; Theor. Chem. Acc. 2016, 135, 151.

45. Grimme, S.; Wiley Interdiscip. Rev. Comput. Mol. Sci. 2011, 1, 211.

46. Grimme, S.; Antony, J.; Ehrlich, S.; Krieg, H.; J. Chem. Phys. 2010, 132.

47. Grimme, S.; J. Chem. Phys. 2006, 124, 034108.

48. Grimme, S.; J. Comput. Chem. 2006, 27, 1787.

49. Albuquerque, A. R.; Garzim, M. L.; Santos, I. M. G. dos; Longo, V.; Longo, E.; Sambrano, J. R.; J. Phys. Chem. A 2012, 116, 11731.

50. Albuquerque, A. dos R.; Estudo Químico-Quântico do Óxido Ti(1-x) CexO2- $\delta$ na Fase Anatase, Universidade Federal da Paraíba, 2014.

51. La Porta, F. A.; Andrés, J.; Vismara, M. V. G.; Graeff, C. F. O.; Sambrano, J. R.; Li, M. S.; Varela, J. A.; Longo, E.; J. Mater. Chem. C 2014, 2, 10164.

52. Kokalj, A.; J. Mol. Graph. Model. 1999, 17, 176.

53. Heifets, E.; Eglitis, R. I.; Kotomin, E. A.; Maier, J.; Borstel, G.; Surf. Sci. 2002, 513, 211.
54. Maul, J.; Santos, I. M. G.; Sambrano, J. R.; Erba, A.; Theor. Chem. Acc. 2016, 135,1 .

55. Oliveira, L. H.; Ramírez, M. A.; Ponce, M. A.; Ramajo, L. A.; Albuquerque, A. R.; Sambrano, J. R.; Longo, E.; Castro, M. S.; La Porta, F. A.; Mater. Res. Bull. 2017, 93, 47.

56. Mazini, M. C.; Sambrano, J. R.; Cavalheiro, A. A.; Leite, D. M. G.; da Silva, J. H. D.; Quim. Nova 2010, 33, 834.

57. Berger, D.; de Moura, A. P.; Oliveira, L. H.; Bastos, W. B.; La Porta, F. A.; Rosa, I. L. V.; Li, M. S.; Tebcherani, S. M.; Longo, E.; Varela, J. A.; Ceram. Int. 2016, 42, 13555.

58. Bradley, C. J.; Cracknell, A. P.; The Mathematical Theory of Symmetry in Solids; Oxford University Press: Oxford, 1972.

59. Suresh, S.; Deepak, T. G.; Ni, C.; Sreekala, C. N. O.; Satyanarayana, M.; Nair, A. S.; Pillai, V. P. P. M.; New J. Chem. 2016, 40, 6228.

60. Mickova, I.; Croat. Chem. Acta 2010, 83, 113. 